\title{
Recent experience with 89 pancreas transplants at a single institution
}

\author{
D. E. R. Sutherland, F. C. Goetz and J.S. Najarian \\ Departments of Surgery and Medicine, University of Minnesota, Minneapolis, Minnesota, USA
}

\begin{abstract}
Summary. Of 89 pancreas transplants performed in 77 diabetic patients ( 43 with and 34 without previous kidney transplants), 53 were from cadaver and 36 from related donors. To date, 64 patients $(83 \%)$ are alive and $27(35 \%)$ have functioning grafts (14>1 year), including 0 out of 3 duct-ligated, 3 out of 15 open-duct, 17 out of 32 enteric-drained, and 7 out of 39 duct-injected. Of technically successful allografts, 8 out of $16(50 \%)$ in the azathioprine- and 17 out of $47(36 \%)$ in the cyclosporin-treated recipients are functioning (eight cyclosporin patients also take azathioprine). Seven of the nine (78\%) non-kidney-transplant recipients of technically successful pancreas allografts from HLA-identical siblings have functioning grafts. Causes of graft failure include allograft rejection, fibrosis secondary to duct injection, or selective $\beta$-cell destruction independent of rejection. Of the 24 recipients who are currently insulin-independent, 14 have normal or nearnormal glucose tolerance test results, while 10 have abnormal results, even though they are otherwise euglycaemic. The patient population to whom pancreas transplantation is applied is gradually changing, and non-uraemic, non-kidney-trans-
\end{abstract}

plant patients currently comprise the majority of our cases (17 out of 24 in 1983; nine of the 17 currently have functioning grafts). We now prefer the enteric drainage technique. Except for recipients of related grafts from a previous kidney donor, in which case it is necessary only to continue the current immunosuppressive regimen, we now administer cyclosporin and prednisone for immunosuppression in recipients of HLAidentical sibling grafts, and cyclosporin, azathioprine and prednisone (triple therapy) for recipients of HLA-mismatched grafts. The most interesting features in this series of cases are the variable patterns of glucose metabolism post-transplant, the finding that processes other than graft rejection, may lead to loss of $\beta$-cell function, preliminary observations on changes in morphology of kidneys following restoration of normoglycaemia, and the evolution of an immunosuppressive regimen that appears to prevent allograft rejection in non-uraemic, non-kidney-transplant patients.

Key words: Pancreas transplant, cyclosporin, azathioprine, HLA-identical siblings, glucose metabolism.
The first clinical pancreas transplant was performed at the University of Minnesota in 1966 [1]. By 1973, Lillehei et al. had placed pancreas grafts in 14 diabetic patients [2]. Between 1974 and 1981, 20 islet transplants were performed in 15 diabetic patients but no recipient became insulin-independent [3, 4]. In July 1978, a new clinical trial of pancreas transplantation was begun [5] with 89 pancreas transplants performed in 77 diabetic patients by March 1984 . Some technically successful grafts have failed for reasons other than rejection [6] and the original disease may recur in the transplanted pancreas. The outcome in the cases since 1978 is summarized.

\section{Subjects and methods}

\section{Subjects}

The 89 pancreas transplants were performed in 77 patients with insulin-dependent diabetes between 25 July 1978 and 7 March 1983. Fiftythree of the pancreases were from cadaver donors ( 32 segmental,
21 whole-organ grafts), and 36 (all segmental) from related donors (5 parents, 17 HLA identical and 7 non-identical siblings, 6 identical twins, 1 cousin). All patients (aged 21-52 years, duration of diabetes 12-34 years) had some evidence of diabetic retinopathy, nephropathy and neuropathy; $96 \%$ of the 71 who were HLA-DR-typed were positive for DR3, DR4 or both.

All of the grafts from related and 25 of the grafts from cadaver donors were of standard segmental (tail) type; five cadaveric grafts were extended segmental (tail and part of head) [7]; two cadaveric grafts included the head and part of the body; and 21 cadaveric grafts were whole pancreas as previously described $[6,7]$. Pancreatic ducts were left open to drain into the peritoneal cavity in 15 cases; 39 ducts were injected (4 with prolamine, 35 with silicone rubber); three ducts were ligated; and 32 ducts were drained into the intestine (pancreatico-jejunostomy). The pancreas grafts from related donors were transplanted immediately, as were 26 grafts from cadaver donors, while 27 were stored at $4{ }^{\circ} \mathrm{C}$ for $1-26 \mathrm{~h}$ before transplantation $[8,9]$. The first 14 recipents of pancreas allografts (June 1978 to August 1980) were immunosuppressed with azathioprine, anti-thymocyte globulin and prednisone. From October 1980 to March 1984, azathioprine was used for 17 and cyclosporin for 44 allograft recipients, with prednisone; and eight recent patients received 'triple therapy' with azathioprine, cyclosporin and prednisone. Five of six recipients of grafts from identical twins received no prophylactic immunosuppression, and one received azathioprine alone. 
Table 1. Outcome after pancreas transplantation at University of Minnesota according to technique, donor source, immunosuppression and recipient renal status

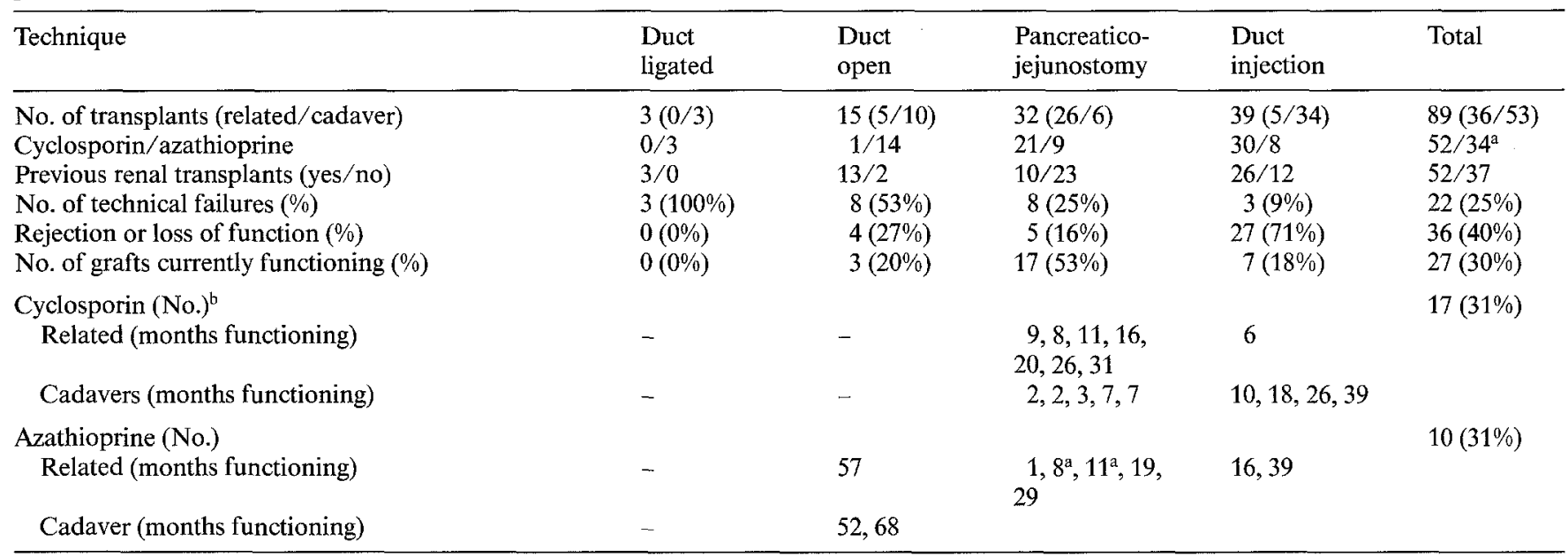

${ }^{a}$ In recipients of pancreas isografts from identical twins, one (now functioning 8 months) was started on azathioprine after insulitis occurred, and the other ( 5 months) received azathioprine from the time of transplantation. One other identical twin received anti-lymphocyte globulin and cyclosporin after insulitis occurred, but became insulin-dependent in spite of the treatment. The other three recipients of isografts (currently not functioning) from identical twin donors were not immunosuppressed ( 2 technical failures, 1 recurrence of hyperglycaemia with insulitis at 6 weeks). ${ }^{b}$ Includes eight patients treated with combination of cyclosporin and azathioprine

\section{Results}

Of the 77 patients transplanted since $1978,64(83 \%)$ are currently alive (March, 1984) and 24 (32\%) insulin-independent at month $1,2,2,3,4,7,7,8,8,10,11,11,13,16$, $16,18,20,26,29,39,39,52$, and 68 after transplantation. Three other patients have functioning grafts (serum and urinary C-peptide above baseline; non-ketosis-prone) at month 6,31 and 57 after transplantation, but take exogenous insulin intermittently for post-prandial hyperglycaemia. The one-year actuarial graft survival rate is $27 \%, 40 \%$ from related and $20 \%$ from cadaver donors. The one-year actuarial patient survival rate is $85 \%$.

The grafts that ceased to function did so for a variety of reasons, including rejection, recurrent $\beta$-cell destruction, possibly fibrosis in some of the duct-injected cases, and technical reasons (Table 1). Thirteen patients died (five with functioning grafts), six from infections, four from cardiovascular disease, one from hyperkalaemia, one from an anaphylactic reaction to anti-lymphocyte globulin, and one for unknown reasons (sudden death at home).

Of the 63 technically successful pancreas allografts, 8 out of $16(53 \%)$ in the azathioprine-treated and 17 out of $47(36 \%)$ in the cyclosporin-treated recipients are functioning [10]. In patients who had not previously received a kidney from the related donor, 7 out of $9(78 \%)$ technically successful grafts from HLA-identical siblings are functioning (the two failures include: recurrence of disease without rejection [6] and one rejection following conversion to azathioprine because of cyclosporin nephrotoxicity). Of five allograft recipients of pancreases without kidney transplantation from HLA non-identical related donors, one treated with azathioprine and three treated with cyclosporin rejected the grafts, while one treated with cyclosporin plus azathioprine (triple therapy) has a functioning graft. Grafts from cadaver donors are functioning in 2 out of $9(22 \%)$ azathioprine-treated and 9 out of $34(26 \%)$ cyclosporintreated recipients.

Of the 27 patients with pancreas grafts that are currently functioning, 24 are insulin-independent. Of these, 14 have normal or nearly-normal glucose tolerance [for method, 11]. The others have glucose tolerance test results that could be classified as either impaired or diabetic. Examples of the ranges of glycaemic excursions between different patients are given in Figure 1. The majority of recipients are euglycaemic and have normal glucose tolerance, as exemplified by the patient (Fig. 1A, 1 year after receiving pancreas graft from sister, duct drainage into Roux-en-y pancreatic jejunostomy).

The follow-up time is too short to make definitive statements as to the effect of pancreas transplantation on secondary complications. Serial biopsies of a prior renal transplant in the patient with the longest functioning pancreatic graft ( $>5$ years) have shown a decrease in glomerular mesangium compared with that present before pancreatic transplant (observations by S.M. Mauer).

Open biopsy of the pancreas graft is necessary to distinguish rejection from other causes of hyperglycaemia in pancreas transplant recipients. The grafts are placed in the peritoneal cavity, so biopsy requires a laparotomy, but we have had no morbidity or complications from the procedure. A wedge of tissue, or a core obtained by a needle inserted into the pancreas under direct vision, or both, are examined histologically. The presence of vasculitis is the only certain criterion for rejection [12]. The presence of insulitis does not necessari- 

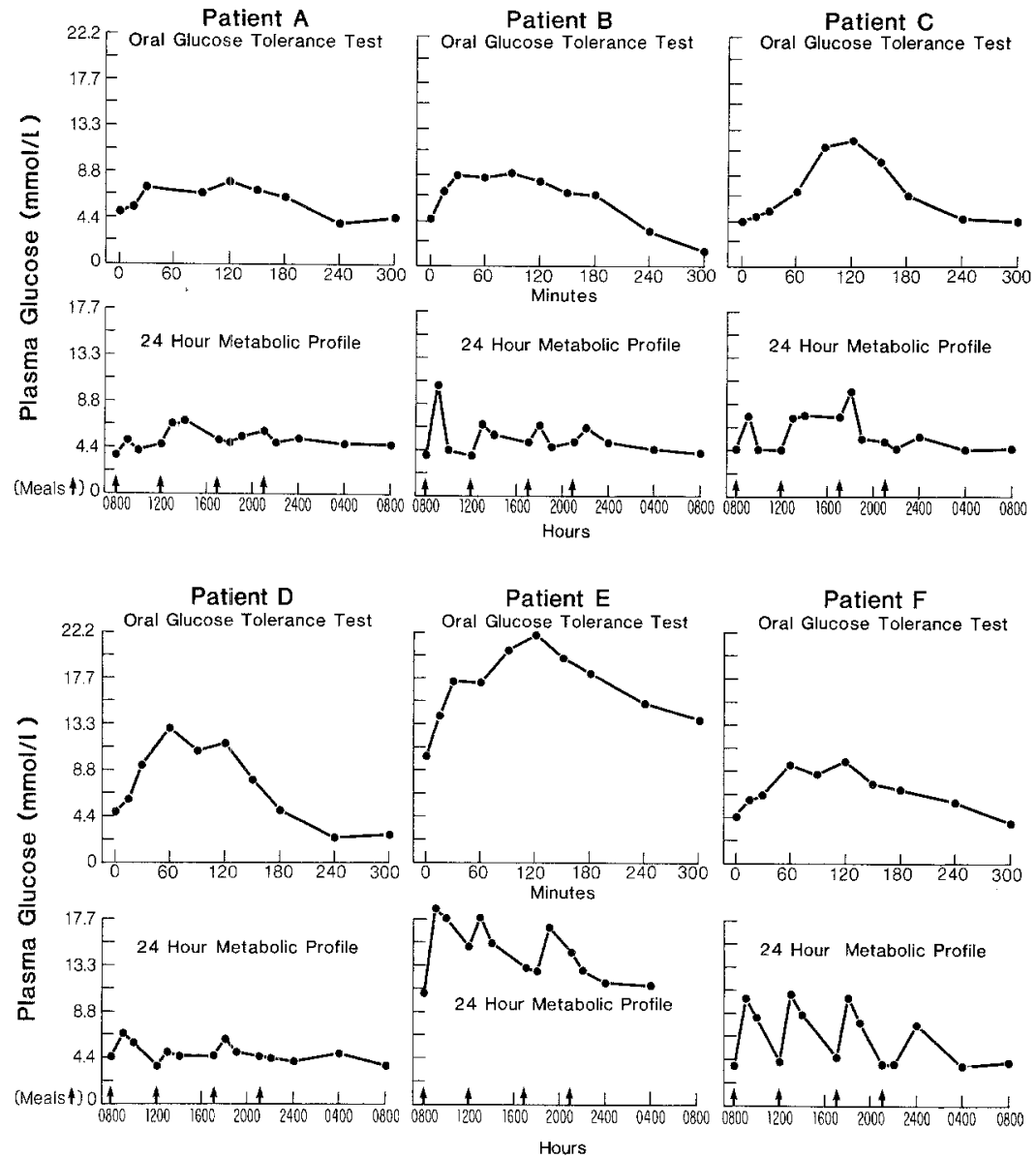

Fig. 1. Results of glucose tolerance tests and 24-h metabolic profiles in the absence of exogenous insulin in six recipients of segmental pancreas transplants, illustrating the variability in response of individual patients. Patient (A), 24 months post-transplant, has both normal glucose tolerance test results and a normal metabolic profile during a day of standard meals and activity. Patient (B), 36 months post-transplant, is normal except for a hypoglycaemic trend at the end of the glucose tolerance test. Patient (C), 6 months post-transplant, has an abnormal (hyperglycaemic) glucose tolerance test result, but is euglycaemic through a day of normal meals and activity. Patient (D), 24 months post-transplant, has an abnormal glucose tolerance test (both hyperglycaemia and hypoglycaemia), but has a normal metabolic profile. Patient (E), 36 months post-transplant, has a very abnormal glucose tolerance test in addition to fasting and post-prandial hyperglycaemia during a profile. Patient (F), 12 months post-transplant, has a normal glucose tolerance test result, but an abnormal metabolic profile with post-prandial hyperglycaemia even though fasting plasma glucose levels are normal ly indicate rejection, since we have seen this feature approximately 6-8 weeks after transplant in three recipients of pancreas isografts from non-diabetic identical twin donors, a finding which probably represents an autoimmune re-enactment of the process that originally destroyed the $\beta$-cells in the native pancreas. Insulitis was associated with recurrence of hyperglycaemia. In one isograft recipient, an insulin-independent state was re-established after treatment with anti-lymphocyte globulin. The insulitis probably resolves after complete selective destruction of $\beta$-cells, an interpretation we currently favour as the explanation for the histological appearance in a case of recurrence of disease in a pancreas allograft recipient previously described [6].

The living related donors for segmental (hemipancreas) transplants underwent several tests of pancreatic function pre- and post-operatively [13]. Of 36 donors, four had abnormal glucose tolerance tests after donation. The others have had normal fasting and 2-h postprandial plasma glucose levels and normal glucose tolerance test results, although sometimes different from those obtained pre-operatively [13]. Exocrine function has also remained normal after donation. Operative complications following hemipancreatectomy have occurred in three donors $(8 \%)$ of whom two required reoperation (one to remove an infarcted spleen, the only splenectomy in the series; and one to religate the pan- creatic duct at the site of transections); the other patient developed a pseudocyst that disappeared and did not recur following percutaneous aspiration.

\section{Discussion}

We have applied pancreas transplantation since 1978 as a therapeutic procedure in patients with diabetes mellitus [5]. The current surgical techniques, methods of immunosuppression, and criteria for patient selection are different from those employed in the earlier series (1966-1973) of Lillehei et al. [1] and represent an evolution in our approaches to pancreas transplantation based on our own experience during the past 5 years [6].

Initially, we performed pancreas transplants in patients who had previously received kidney transplants [5]. In 1980 we began to accept for pancreas transplantation non-uraemic, non-renal transplant patients, whose complications of diabetes were more severe than the potential side effects of immunosuppression. Of 32 patients in this category, 13 (41\%) currently have functioning grafts. Such patients now constitute the majority of our pancreas transplant recipients (17 of 24 in 1983; 9 of the $17(53 \%)$ currently have functioning grafts). All of the patients have had some evidence of diabetic ne- 
phropathy, either functionally and morphologically, or morphologically alone on kidney biopsy.

In 1979, we began to accept living related donors for transplantation, and $36(40 \%)$ of our transplants have been from this source. Grafts from related donors appear to be less likely to be rejected. In our total experience, 16 out of $36(44 \%)$ of grafts from related and 11 out of $53(21 \%)$ of grafts from cadaver donors are currently functioning (of transplants done in 1983, 4 out of $14(29 \%)$ from cadaver and 7 out of $10(70 \%)$ from related donors are functioning). The surgical complication rate in related pancreas donors has been lower than in our related kidney donors. However, four pancreas donors $(11 \%)$ have had abnormal changes in glucose tolerance. Thus, we need to develop tests to predict the potential changes that will follow hemipancreatectomy. The best results are in the situation where the recipient has previously received a kidney graft from the same donor (10 cases); 5 out of 6 technically successful grafts $(83 \%)$ in this category are functioning. In the recipients of related grafts who have not had previous kidney transplants ( 24 cases), 10 out of 17 technically successful grafts are functioning (59\%), including 7 out of 9 (78\%) allografts from HLA-identical sibling and 2 out of $3(67 \%)$ isografts from identical twin donors.

We have used a variety of techniques for pancreas transplantation. The open duct method (15 cases) has been associated with three long-term functioning grafts (4.4 to 5.6 years), but four ( $26 \%$ ) of the recipients developed severe ascites and we thus no longer use this technique. Three duct-ligated grafts failed, but none of the failures ( 1 due to bleeding, 1 to chemical peritonitis and 1 to inadequate preservation) could be related to the method. The duct injection technique, adopted in 1980 , has been used in 39 cases, primarily for recipients of cadaver pancreas transplants; the increased propensity to rejection of cadaver grafts may be responsible for the low success rate of this technique in relation to the outcome of pancreas grafts anastomosed to a Roux-en-Y limb of recipient jejunum. The pancreaticoenterostomy technique, introduced in 1981, primarily has been used in recipients of grafts from related donors (12 out of 26 functioning, $46 \%$ ), but recently we have used this method in recipients of grafts from cadaver donors as well ( 6 cases, 5 currently functioning, $83 \%$ ). We place a catheter in the pancreatic duct, but leave it within the bowel and we do not bring it externally, in contrast to the practice of other groups $[14,15]$. Two anastomotic leaks have occurred (the second and sixth cases of enteric drainage); the other six technical failures were from vascular thrombosis. Our current approach is to perform a Roux-en-Y graft pancreaticojejunostomy in all recipients. The whole pancreas technique (without the duodenum) cannot be used for transplantation from related donors [7] but has been used for 21 pancreas transplants from cadaver donors (15 with silicone rubber injection, 1 open duct and 5 with anastomosis of the papilla of Vater to a Roux-en-Y limb of recipient jeju- num), of which $6(29 \%)$ are functioning (compared with 5 out of 32 segmental cadaver grafts, $16 \%$ ). The putative advantages of the whole pancreas technique include a lesser propensity to vascular thrombosis and a larger number of islets, resulting in a greater reserve (compared with segmental grafts) for residual function should fibrosis or rejection episodes reduce the $\beta$-cell mass.

With regard to immunosuppression and pancreas graft survival rates (for technically successful grafts), $50 \%$ of azathioprine- and $32 \%$ of cyclosporin-treated recipients have functioning grafts, but six of the azathioprine-treated patients were recipients of pancreas grafts from the same related donor as had provided a previous kidney graft, and it was then only necessary to continue whatever immunosuppressive regimen had been sufficient to prevent rejection of the kidney. Good graft survival rates are also associated with transplantation of hemi-pancreases from HLA-identical siblings to cyclosporin-treated recipients. The survival rates of technically successful grafts from cadaver donors is low in both cyclosporin- ( 9 out of $34,26 \%$ currently functioning) and azathioprine-treated recipients ( 3 out of 10 , $30 \%$ currently functioning) and it is only recently that pancreas graft function has been successful for more than a few months in non-uraemic, non-kidney-transplant recipients of cadaver grafts. Our current protocol for such patients is: (1) to administer blood transfusion before transplant while on immunosuppression; (2) to perform splenectomy; (3) to administer cyclosporin-anti-lymphocyte globulin-prednisone for post-transplant immunosuppression and to add azathioprine after the anti-lymphocyte globulin course; (4) to biopsy the graft if plasma glucose levels increase over baseline; and (5) to administer another course of anti-lymphocyte globulin if a biopsy shows features consistent with rejection.

The most interesting findings in our pancreas transplant recipients are: (1) the extreme variations in plasma glucose levels and results of glucose tolerance tests in the patients with functioning grafts [13]; and (2) the finding on graft biopsies that processes other than allograft rejection or graft fibrosis may lead to islet dysfunction or loss of islet function and recurrence of diabetes [6]. With regard to the first observation, patients with Type 1 diabetes may be insulin-independent after pancreas transplantation, but metabolic abnormalities may persist, possibly because Type 2 (non-insulin-dependent) diabetes may also have been present and simply masked pre-transplant because of co-existing Type 1 diabetes.

With regard to the biopsy findings in recurrent diabetes, two patients (both insulin-independent) with impaired glucose tolerance tests post-transplant had increased numbers of somatostatin-positive cells within the graft islets of Langerhans. Finally, three out of four recipients of grafts from identical twin donors developed insulitis, a process that was abated in one with antilymphocyte globulin treatment. The latter findings are 
consistent with an autoimmune aetiology of diabetes, at least in these particular cases, and will be reported in detail elsewhere.

Recurrence of disease obviously is not inevitable in pancreas transplant recipients, since 14 of our recipients currently have grafts functioning for more than one year, the longest over 5 years. The objective of pancreas transplantation, to prevent or halt the progression of secondary complications in selected diabetic patients, should therefore be feasible.

Acknowledgements. This study is supported in part by NIH grants AM19269, AM13083 and RR 400 .

\section{References}

1. Lillehei RC, Ruiz JO, Acquino C, Goetz FC (1976) Transplantation of the pancreas. Acta Endocrinol 83 (Suppl 205): 303-320

2. Sutherland DER, Goetz FC, Carpenter AM, Najarian JS, Lillehei RC (1974) Pancreaticoduodenal grafts: clinical and pathological observations in uremic versus non-uremic recipients. In: Touraine JL, Traeger J, Beutel H (eds) Transplantation and clinical immunology, Vol.X. Excerpta Medica, Amsterdam, pp 190-195

3. Najarian JS, Sutherland DER, Matas AJ, Steffes MW, Goetz FC (1977) Human islet transplantation: a preliminary experience. Transpl Proc 9: 233-236

4. Sutherland DER, Matas AJ, Goetz FC, Najarian JS (1980) Transplantation of dispersed pancreatic islet tissue in humans: autografts and allografts. Diabetes 29 (Suppl 1): 31-44

5. Sutherland DER, Goetz FC, Najarian JS (1979) Intraperitoneal transplantation of immediately vascularized segmental pancreatic grafts without duct ligation: a clinical trial. Transplantation 28 : 485-491

6. Sutherland DER, Goetz EC, Elick BA, Najarian JS (1982) Experience with 49 segmental pancreas transplants in 45 diabetic patients. Transplantation $34: 330-338$
7. Sutherland DER, Chinn PL, Elick BA, Najarian JS (1984) Maximization of islet mass in pancreas grafts by near total or total whole organ excision without the duodenum from cadaver donors. Transpl Proc 16: 115-119

8. Florack G, Sutherland DER, Heil J, Zweber N, Najarian JS (1982) Long-term preservation of segmental pancreas autografts. Surgery 92: $260-269$

9. Florack G, Sutherland DER, Chinn PL, Najarian JS (1984) Clinical experience with transplantation of hypothermically preserved pancreas grafts. Transpl Proc 16: 111-114

10. Sutherland DER, Chinn PL, Goetz FC, Elick BA, Najarian JS (1983) Experience with cyclosporin versus azathioprine for pancreas transplantation. Transpl Proc 15: 2602-2612

11. Sutherland DER, Najarian JS, Greenberg BZ, Senske BJ, Anderson GE, Francis RS, Goetz FC (1981) Vascularized segmental transplantation on the pancreas in insulin-dependent patients: Hormonal and metabolic effects of an endocrine graft. Ann Int Med 95: 537-541

12. Sibley RK, Mukai K (1983) Pathological features in 29 segmental pancreas transplants in 27 diabetic patients. Lab Invest 48: 78 ( $\mathrm{Ab}$ stract)

13. Chinn PL, Sutherland DER, Goetz FC, Oliphant UJ, Elick BA, Najarian JS (1984) Metabolic effect of hemipancreatectomy in living related graft donors. Transpl Proc 16: 11-17

14. Groth CG., Collste H, Lundgren G (1982) Successful outcome of segmental human pancreatic transplantation with enteric diversion after modification in technique. Lancet $2: 522-524$

15. Calne RY, White DJG, Rolles K, Duffy TJ, Kass T (1982) Renal and segmental pancreatic grafting with drainage of exocrine secretion and initial continuous intravenous cyclosporin $\mathrm{A}$ in a patient with insulin-dependent diabetes and renal failure. $\mathrm{Br}$ Med J 285: $677-680$

Dr. D. Sutherland

Box 280

University of Minnesota Hospitals

420 Delaware Street SE

Minneapolis, MN 55455

USA 